\title{
Télécommunications Et Croissance Économique Au Bénin
}

\section{Sévérin Bokini}

Faculté des Sciences Economiques et de Gestion (FASEG)

Université de Parakou, Bénin

Doi:10.19044/esj.2019.v15n13p400 URL:http://dx.doi.org/10.19044/esj.2019.v15n13p400

\section{Resume}

Cet article examine la relation entre les télécommunications et la croissance économique au Bénin. A partir de la technique de l'économétrie des séries temporelles et avec un modèle à correction d'erreur à la Engle et Granger, sur la période de 1980 à 2017, l'article analyse la nature de la corrélation qui existe entre la croissance économique et le niveau de développement des télécommunications (mesuré par le taux de pénétration mobile) au Bénin. Les résultats montrent qu'il existe une relation positive aussi bien à court terme qu'à long terme entre le taux de pénétration mobile dans le secteur du numérique et le PIB réel. En outre, l'analyse de la causalité au sens de Granger révèle l'existence d'une relation bidirectionnelle et permet d'affirmer que le taux de pénétration mobile favorise la croissance économique et la croissance économique aussi à son tour favorise le taux de pénétration mobile. Autrement dit, le développement des télécommunications représente pour le Bénin une opportunité de croissance économique durable tout comme une accélération du taux de croissance améliore le taux de pénétration mobile.

Mots clés : croissance économique, Technologie de l'Information et de la Communication, taux de pénétration mobile, causalité, Bénin 


\title{
Telecommunications and Economic Growth in Benin
}

\author{
Sévérin Bokini \\ Faculté des Sciences Economiques et de Gestion (FASEG) \\ Université de Parakou, Bénin
}

\begin{abstract}
This article examines the relationship between telecommunications and economic growth in Benin. Using the time series econometric technique and with an Engle and Granger error correction model over the period from 1980 to 2017 , the article analyzes the nature of the correlation between economic growth and the level of telecommunications development (measured by penetration rate) in Benin. The results show that there is a positive relationship both in the short and long term between the mobile penetration rate in the digital sector and real GDP. In addition, the Granger causality analysis reveals the existence of a bidirectional relationship and asserts that the mobile penetration rate promotes economic growth and economic growth also in turn promotes the mobile penetration rate. In other words, the development of telecommunications represents for Benin an opportunity for sustainable economic growth just as an acceleration of the growth rate improves the mobile penetration rate.
\end{abstract}

Keywords: Economic growth, Information and Communication Technologies, mobile penetration rate, Causality, Benin

\section{1- INTRODUCTION}

Les Télécommunications et les Technologies de l'Information et de la Communication (TIC) ont partout montré leurs preuves comme facteurs incontournables de développement et un secteur transversal à effet multiplicateur direct sur l'ensemble des autres secteurs de l'activité économique. Elles constituent également un moyen de renforcement des capacités, que ce soit en milieu d'affaires ou dans les zones rurales ou isolées (INSAE, 2010). L'existence d'un réseau de télécommunication performant constitue pour l'économie nationale un facteur de développement. Reconnaissant l'importance de la communication, l'assemblée générale des Nations Unies proclame 1983 comme année de la communication (Arlandis, 1987). Ce faisant, elle exprime sa conviction que l'événement fournirait à tous les pays l'occasion d'analyser en profondeur leur politique en matière de 
développement des communications, et stimulerait en particulier le secteur des infrastructures de télécommunications. Malheureusement, l'environnement africain de l'information et des télécommunications présente les caractéristiques suivantes : faible taux de pénétration de la téléphonie, faible croissance de réseaux, tarification élevée des installations privées, liaisons téléphoniques interurbaines médiocres et infrastructures de réseaux nationaux très variables (ECA, 1999). En effet, « la modernisation des infrastructures de télécommunications constitue un enjeu stratégique majeur pour le développement de toutes les grappes, en particulier celle de «transports, logistique et commerce» tournant autour des activités portuaires. Elles offrent, de façon spécifique, la possibilité de s'approprier le savoir et le savoir-faire nécessaire au développement humain durable » (INSAE, 2010 ; p4). Dans la problématique de l'accélération de la croissance économique, les télécommunications sont créditées d'un caractère transformateur et induisent des changements profonds et significatifs (Youssef et M'Henni, 2004).

Des études mettant en exergue l'impact du développement des télécommunications sur la croissance économique dans les économies développées ont conclu à des relations positives entre télécommunications et croissance économique (Jipp, 1963; Hardy, 1980; Moss, 1981; Saunders et al., 1994; Lichtenberg, 1995; Greenstein et Spiller, 1996). Cependant, peu de travaux de recherche ont été menées sur ce sujet en Afrique subsaharienne. En outre, les études ont examiné la relation entre les télécommunications et la croissance économique sans prendre en compte l'importance (l'ampleur) de son impact sur la croissance économique, ni tenir compte de la relation de causalité entre le développement des télécommunications et la croissance économique. Si le développement des télécommunications s'est avéré comme l'un des facteurs qui puissent influer sur la croissance économique, sa contribution peut varier selon les pays et différents niveaux de développement. De plus, des travaux indiquent que la contribution des télécommunications à la croissance économique est tributaire du niveau de développement des télécommunications (Roller et Waverman, 2001; Karner et Onyeji, 2007). De ce point de vue, la présente recherche apporte une contribution empirique à la recherche économique, non seulement, en examinant la relation entre les télécommunications et la croissance économique mais aussi en questionnant la nature de la causalité entre le développement des télécommunications et la croissance économique dans le cas du Bénin.

En se basant sur le rapport GSMA (2017), les technologies et services mobiles ont généré 110 milliards de dollars de valeur économique en Afrique subsaharienne, soit 7,7\% du PIB en 2016. Les prévisions faites pour l'horizon 2020, rendent compte de ce que la contribution du mobile au PIB passe à 142 milliards de dollars, soit $8,6 \%$ du PIB, puisque les pays bénéficient d'une amélioration de la productivité et de l'efficacité apportées par l'augmentation 
des abonnements aux services mobiles. L'écosystème du secteur mobile a également permis de soutenir 3,5 millions d'emplois en Afrique subsaharienne en 2016. Outre l'impact du secteur du mobile sur l'économie et le marché du travail, il apporte également une contribution importante au secteur public, avec 13 milliards de dollars payés en 2016 sous forme d'impôts.

Par ailleurs, le contexte social, institutionnel, législatif et réglementaire, dans lequel les télécommunications sont introduites et mises en œuvre, est déterminant de leurs impacts sur le développement. En effet, le manque d'organisation et la dégradation de l'environnement réglementaire dans le secteur des télécommunications au Bénin ont occasionné d'importants préjudices à l'économie béninoise (la mauvaise gouvernance enregistrée au niveau de l'opérateur historique qu'est LIBERCOM ${ }^{32}$, le bradage, dès la libération du secteur en 2000, des licences d'exploitation aux entreprises de GSM (entreprise la téléphonie mobile ${ }^{33}$ ) avec un prix de cession de 120 millions ${ }^{34}$ de Fcfa par licence, la crise des entreprises de téléphonie mobile de 2007, la mise en place des pratiques frauduleuses au niveau des appels internationaux par les entreprises de téléphonie mobile). Force est de constater que le développement du secteur des télécommunications n'a pas encore permis d'entraîner une croissance significative au Bénin. En effet, les télécommunications ne représentent que $5,8 \%$ du produit intérieur brut (PIB) en 2014 et en 2015 (ARCEP-BENIN ${ }^{35}$, 2018). Leur contribution à la croissance reste également faible : 3,03 point de pourcentage en 2014 contre 2,05 point de pourcentage en 2013 (ARCEP-BENIN, 2017). Actuellement, le taux de pénétration téléphonique mobile a connu une amélioration et est passé de 76,58\% au 31 décembre 2017 à 81,17\% au 31 mars 2018 environ 5 points de pourcentage. Mais un problème est suscité : celui de la couverture géographique du territoire national voire celui du service universel. En effet, malgré cette amélioration, on note une faible pénétration du téléphone dans les zones rurales.

En effet, le téléphone fixe était le seul moyen de communication à distance depuis les années 1975 au Bénin. Détenu par l'autorité publique, il n'a pas été bénéfique au développement du secteur des télécommunications qui est caractérisé par un faible taux de pénétration de téléphone et des liaisons interurbaines médiocres (Chabossou, 2010). C'est ce qu'a suscité l'arrivée de

\footnotetext{
${ }^{32}$ LIBERCOM est la filiale de Bénin Télécoms SA

${ }^{33}$ La téléphonie mobile désigne indifféremment le téléphone mobile, le téléphone portable ou le téléphone cellulaire ;

${ }^{34}$ Les coûts de la licence GSM dans la sous-région : Togo: 800000 millions pour une durée de 10 ans ; Burkina-Faso: 5,6 milliards pour une durée de 10 ans ; Niger: 4,3 milliards pour une durée de 15 ans ; Côte d'Ivoire: 40 milliards pour une durée de 20 ans ; Mauritanie: 30 milliards pour 20 ans ; Sénégal: 70 milliards pour 20 ans

35 ARCEP-BENIN : Autorité de Régulation des Communications Electroniques et de la Poste du Bénin
} 
la téléphonie mobile au Bénin en 2000, qui aujourd'hui a permis une amélioration de ce secteur.

$\mathrm{Au}$ cours de ces dernières années, l'importance de l'usage du téléphone fixe et mobile n'est plus à démontrer. C'est ainsi, qu'au Bénin, l'ouverture des télécommunications (le téléphone mobile) à la concurrence, à partir des années 2000 a permis aux populations (dans les zones urbaines à celles rurales) d'avoir plus accès à l'information. Cette ouverture à la téléphonie mobile a surtout contribué au désenclavement des zones rurales béninoises et a favorisé l'accès des populations à de nouveaux services. Quant au téléphone fixe (monopole public) qui est confié au Bénin Télécom SA (ex OPT $)^{36}$, il n'a pas été bénéfique au développement de ce secteur caractérisé par un faible taux de pénétration du téléphone et liaisons interurbaines médiocres (Chabossou, 2015). Le Bénin s'est donc engagé dans une politique qui vise la recherche d'une croissance économique pour l'amélioration du bien-être de la population, l'accélération de la lutte contre la pauvreté et l'atteinte des objectifs du document de Stratégie de Croissance et de Réduction de la Pauvreté (SCRP, 2016). Cependant, il ne peut y avoir croissance rapide dans un pays sans une variété d'infrastructures de qualité et de niveau satisfaisant. Les infrastructures téléphoniques jouent un rôle crucial dans le développement économique et social d'un pays (Meleu, 1999). Elles constituent des facteurs de production pour de nombreuses industries et permettent de changer les formes d'accumulation des richesses : réduction du coût d'accès à l'information et amélioration de la coordination des acteurs en dégageant des gains de productivité (économie d'échelle, externalités) pour tout le système productif en ce sens qu'elles jouent le rôle d'accompagnement de la production des autres secteurs. De plus en plus, les infrastructures de télécommunications participent au bien être des consommateurs et incitent aujourd'hui le niveau de consommation des services de télécommunications (Sridhar et Sridhar, 2007). Devant cette nécessité pour tout pays de disposer des télécommunications, notamment des services téléphoniques de base fiables et en quantité suffisante, le Bénin s'est lancé dès 1998 au développement des télécommunications. Cette volonté de maintenir le secteur à la pointe de la technologie est effective et se concrétise entre autre par l'introduction de la téléphonie mobile et un développement de l'accès à l'Internet. Lancé en 1995 avec un opérateur (BENIN TELECOM.SA qui est un monopole), le secteur de la téléphonie mobile en comptait en 2007 cinq opérateurs : LIBERCOM ; MTN BENIN; MOOV BENIN ; BELL BENIN COMMUNICATION et GLO MOBILE BENIN. Après la dissolution de Libercom (le 21 juin 2017) ; la révocation de la convention d'exploitation de Bell Bénin communication (le 02 août 2017) puis le retrait de la licence

\footnotetext{
${ }^{36}$ Office des Postes et Télécommunication
} 
d'exploitation de Glo mobile Bénin (le 18 décembre 2017), aujourd'hui le secteur béninois de télécommunication ne compte que deux opérateurs de téléphonie mobile à savoir : MTN BENIN et MOOV BENIN. Ainsi, au 31 mars 2018, le parc des abonnés actif à la téléphonie s'élève à 9379245 contre 9760940 au 31 mars 2017 (ARCEP-BENIN, 2018); soit une baisse de 03,91\% du parc en espace d'une année dont une des raisons est la dissolution de LIBERCOM et de la disparition de GLOBACOM. Il s'ensuit alors une baisse du taux de pénétration mobile et celle de la télé densité mobile au Bénin qui est passée de $86,91 \%$ en 2015 à 76,58\% en 2017, soit une baisse de près de 10 points. Dans la même période, la télé densité fixe est passée de $1,82 \%$ à $0,48 \%$. Au total, le taux de pénétration téléphonique mobile au 31 mars 2018 est de l'ordre de $81,17 \%$ au Bénin. Malgré cette rapide amélioration du taux de pénétration des réseaux GSM au Bénin, le nombre de ligne pour 100 habitants (télé densité) demeure faible (ARCEP-BENIN, 2017). De même, le volume des investissements a atteint 42,38 milliards de FCFA en 2017 contre 46,45 milliards de FCFA en 2016 soit une baisse de 8,76\%. De plus, à l'échelle mondiale une augmentation de $\$ 1000$ US du PNB par habitant est associée à une augmentation de 2,4 de la télé densité. Ainsi, en 2015, les plus grands opérateurs mondiaux de télécommunications par nombre d'abonnés étaient : 199 millions ; SingTel : 146 millions ; Axiata Group Berhad : 159,7 millions; China Unicom : 169,7 millions; Telia : 160 millions; Saudi Telecom Company : 139 millions; MTN Group : 137,47 millions (ARCEP-BENIN, 2018)). Ce phénomène est souvent signifiant qu'il y a un rapport de causalité qui s'applique dans deux directions : l'amélioration des communications génère une croissance économique qui à son tour déclenche le besoin d'une meilleure infrastructure des communications (Banque mondiale, 1994). Par ailleurs, il faut noter que le Bénin appartient au groupe des pays les moins avancés avec une croissance du PIB réel de 3,5\% en 2012. En progression par rapport aux 4\% de 2016 ce taux est estimé à 5,5\% en 2017 selon le groupe de la Banque Africaine de Développement. Il connait davantage une forte amélioration atteignant $6,5 \%$ en 2018 (BAD, 2018). C'est la preuve d'une progression de l'activité économique. Enfin, la littérature économique sur la relation entre télécommunications et la croissance économique est très peu fournie en ce qui concerne le contexte béninois. Cette recherche contribue à combler ce vide et se propose d'étudier la question centrale suivante : l'existence d'un réseau de télécommunication performant et accessible constitue-t-elle pour l'économie nationale, un facteur de croissance économique ? Cette étude apportera des réponses à cette interrogation tout au long de son développement.

L'objectif de cet article est d'analyser le lien entre les télécommunications et la croissance économique au Bénin. Au plan méthodologique, trois approches sont généralement adoptées pour mettre en 
évidence cette contribution. La première approche permet de faire une comptabilisation de la croissance qui permet de dissocier l'amélioration de la productivité due aux différents facteurs de production. La deuxième approche d'analyse de la contribution des télécommunications à la croissance se fonde sur une logique sectorielle et permet d'évaluer la contribution des différents secteurs de production et d'utilisation des télécommunications à la croissance de la productivité de l'économie. La troisième approche est celle économétrique. Elle est utilisée pour déterminer l'interdépendance entre les variables télécommunications et la croissance économique (Youssef et M'Henni, 2004 ; Chabossou, 2017). Ainsi, l'analyse de la corrélation entre les facteurs télécommunications et l'augmentation de la productivité permet d'identifier les variables qui contribuent à l'augmentation et celles qui n'y contribuent pas. Cette dernière approche est adoptée dans le cadre de cette étude pour analyser la contribution du taux de pénétration mobile à la croissance économique au Bénin.

La suite de cet article est organisée en 4 sections. Dans la section 2, nous présentons une revue de littérature montrant le débat sur le lien entre Télécommunication et croissance économique. Dans la section 3, nous présentons la méthodologie adoptée. Les résultats et leurs discussions sont présentés dans section 4. La conclusion est consacrée à la section 5 .

\section{2- Revue de littérature sur le lien entre Télécommunications et croissance économique}

$\mathrm{Au}$ plan théorique, le changement technologique était considéré comme une variable résiduelle, permettant d'expliquer la croissance (Solow, 1956). De même, les développements de la théorie de la croissance endogène mettent en évidence la dépendance des taux de croissance économique au changement technologique (Romer, 1986). Ainsi, Barro (1990) soutient que les dépenses en infrastructure, entre autres les infrastructures de télécommunication sont productives. Elles engendrent les externalités positives favorables à la croissance économique.

$\mathrm{Au}$ plan empirique, le lien entre télécommunication et croissance économique a été largement étudié par les chercheurs ces dernières années. Cette littérature économique cherche à établir un lien entre les télécommunications et la croissance économique. Par exemple, Ricketts (2002) conclut notamment que les télécommunications facilitent la coordination de la circulation de l'information, offrent des possibilités d'accroissement de l'efficacité de l'interaction et de la coordination et influent de cette manière sur le développement des activités économiques. Les activités économiques nécessitent des niveaux importants d'interaction et de coordination pour pouvoir être menées avec succès et efficacité. Alleman et al. (2014) ont en revanche montré qu'une infrastructure de télécommunication 
moderne est non seulement essentielle à la croissance économique nationale, mais également à la participation à des marchés mondiaux de plus en plus concurrentiels et à la recherche de nouveaux investissements. En règle générale, les télécommunications et leurs auxiliaires contribuent à la croissance économique par les canaux suivants: augmentation de la productivité dans tous les secteurs; facilitation de l'expansion du marché audelà des frontières pour réaliser des économies d'échelle; réduction des coûts des services en facilitant l'accès, notamment dans les domaines de l'administration, de l'éducation, de la santé et des services bancaires; facilite l'accès à la recherche; développement de produits et services TIC; contribution à une meilleure gouvernance, préalable à la croissance, par une participation, une responsabilisation et une transparence accrues.

L'utilisation des services de télécommunication génère des externalités positives en améliorant la créativité, l'apprentissage et la résolution de problèmes. Son impact à court terme se fait sentir sur l'emploi tandis qu'à long terme, il se manifeste par l'interaction entre connectivité, accès, sécurité du réseau, capacités et/ou compétences, structures de marché et gouvernance d'entreprise, ainsi que de l'environnement réglementaire (ATCI $\left.{ }^{37}, 2009\right)$. Cela détermine certainement si les entreprises des pays en développement peuvent participer de manière effective et efficace à l'économie de l'information et être concurrentielles sur les marchés électroniques mondiaux.

Par exemple, Karner et Onyeji (2007) examinent la contribution des investissements privés en télécommunications dans la croissance économique de 14 pays africains et 13 pays d'Europe du centre et orientale (PECO) pour la période allant de 1999 à 2005 . Leurs résultats montrent que la contribution est positive, mais non significatif. Selon eux, cela pourrait être dû au niveau relativement bas d'infrastructures de télécommunications dans les pays sélectionnés, ce qui réduit l'efficacité des investissements privés dans les télécommunications.

Dans une autre dimension, Roller et Waverman (2001) ont examiné l'impact de l'investissement dans les infrastructures de télécommunication sur le PIB de 21 pays de l'OCDE et de 14 pays non membres de l'OCDE en développement ou nouvellement industrialisés entre 1970 et 1990 . Les auteurs ont constaté que cet impact n'est pas linéaire : il est plus élevé dans les pays de l'OCDE que dans les pays non membres et dans les pays ayant atteint la «masse critique», c'est-à-dire que le nombre de lignes téléphoniques principales dépasse 40 pour 100 personnes.

De même, Sridhar et Sridhar (2007) ont étudié la relation entre les télécommunications et la croissance économique à l'aide de données

${ }^{37}$ ATCI : Agence des Télécommunications de Côte d’Ivoire 
provenant de 28 pays en développement. L'étude révèle que les lignes fixes ont un impact positif et que la pénétration du téléphone mobile a un impact significatif sur la production nationale. L'impact de la pénétration des télécommunications sur la production totale s'avère nettement plus important pour les pays en développement que pour les pays de l'OCDE. Cela corrobore avec les résultats d'une étude de Waverman et al. (2005) qui ont constaté que la téléphonie mobile avait un impact positif et significatif sur la croissance économique.

Pour aller plus loin, Cronin et al. (1993) ont examiné le lien de causalité entre les télécommunications et la croissance économique. L'étude a révélé une relation bidirectionnelle entre l'infrastructure de télécommunication et la croissance économique aux États-Unis. Une analyse de causalité réalisée par Madden et Savage (1998) confirme également une relation bidirectionnelle entre l'investissement en télécommunications et la croissance économique dans les pays d'Europe du centre et orientale. L'étude de Chakraborty et Nandi (2003) a montré une relation bidirectionnelle entre la télédensité et le Produit Intérieur Brut (PIB) à court et à long terme dans 12 pays en développement d'Asie. Lorsque ces pays sont divisés en deux groupes avec un degré de privatisation élevé et faible, la causalité n'est bidirectionnelle que pour les pays du premier groupe. Cieslika et Kaniewsk (2004) confirment l'existence d'un lien de causalité positif et statistiquement significatif entre l'infrastructure de télécommunication et les revenus au niveau régional en Pologne et conclut que la causalité va du premier au second. Yoo et Kwak (2004) ont constaté une relation bidirectionnelle entre les investissements dans les technologies de l'information et de la communication et la croissance économique en Corée du Sud sur la période 1965-1998. Une étude plus récente réalisée par Wolde-Rufael (2007) révèle également une relation bidirectionnelle entre les deux aux États-Unis entre 1947 et 1996.

Cependant, Shiu et Lam (2008) ont constaté une relation unidirectionnelle entre le développement du PIB et le développement des télécommunications en Chine. La causalité dans la direction opposée, c'est-àdire des télécommunications à la croissance économique, ne se rencontre que dans la riche région orientale, mais pas dans les provinces à faible revenu du centre et de l'ouest.

Selon Qiang (2009), dans une analyse récente de la Banque mondiale visant à tester l'impact du taux de pénétration des télécommunications sur les taux de croissance économique au niveau de 120 pays, montre que l'accroissement du taux de pénétration des téléphones mobiles de $10 \%$, entraîne une augmentation de la croissance économique de $0,81 \%$ dans les pays en développement, contre $0,60 \%$ dans les pays développés. L'étude a également révélé que toutes les technologies de l'information et de la communication promeuvent la croissance plus efficacement dans les pays en 
développement que dans les pays développés. En effet, les services de télécommunication contribuent à améliorer le fonctionnement des secteurs public et privé. Les problèmes étaient plus graves dans les pays en développement que dans les pays développés. Par conséquent, les pays en développement gagnent plus en résolvant certains d'entre eux grâce à un meilleur accès aux télécommunications (Qiang, 2009). Il ressort clairement de cette littérature que peu d'efforts ont été faits dans les travaux antérieurs pour examiner le lien entre le développement des télécommunications et la croissance économique des économies africaines. C'est l'une des principales motivations de ce papier.

\section{3- Méthodologie}

Pour l'évaluation empirique de la relation entre les télécommunications et la croissance économique au Bénin, nous avons choisi une fonction de production néoclassique de type Cobb-Douglas. La fonction de production néoclassique de type Cobb-Douglas standard et homogène se présente comme suit :

$Y_{t}=A K^{\alpha} L^{\beta}$

On remplace K par le stock de capital investi dans le TIC (INVTIC), et $\mathrm{L}$ par le taux brut de scolarité secondaire (TBSS). Dans cette expression A représente la productivité globale des facteurs dont la variation relative (le progrès technique) représente la partie de la croissance de la production non expliquée par les montants de capital et de travail. En effet, à la suite des développements de la théorie de la croissance endogène mettant en évidence la dépendance des taux de croissance économique au changement technologique (Romer, 1986) et de Barro (1990) qui soutient que les dépenses en infrastructure, entre autres les infrastructures de télécommunication, engendrent des externalités positives favorables à la croissance économique, nous introduisons dans le modèle la variable taux de pénétration mobile qui correspond au nombre d'abonnés locaux à la ligne téléphonique fixe et au téléphone mobile pour mille habitants. L'équation (1) devient:

$Y_{t}=A . I N V T I C^{\alpha} T_{B S S}^{\beta} T P M^{\gamma}$

Dans cette fonction de production néoclassique de type Cobb-Douglas, Y représente le PIB qui exprime la production totale ou le Produit Intérieur Brut. Le produit intérieur brut est exprimé en fonction du taux de pénétration mobile (TPM), du stock du capital humain qui est représenté par le taux brut de scolarisation secondaire (TBSS), du stock de capital investi dans les TIC (INVTIC) et des autres facteurs (A) qui influencent le produit national et qui sont observés à chaque période de temps t. En tenant compte des variables explicatives sus indiquées et après linéarisation de l'équation (2), nous obtenons la forme linéaire du modèle empirique final qui prend cette forme: 


$$
\operatorname{Ln}\left(P I B_{t}\right)=\eta+\alpha \operatorname{Ln}\left(\text { INVTIC }_{t}\right)+\beta \operatorname{Ln}\left(\text { TBSS }_{t}\right)+\gamma \operatorname{Ln}\left(T P M_{t}\right)+\varepsilon_{t}
$$

Où : $L n$ représente le logarithme népérien ; $\alpha_{i}$ sont des paramètres à estimer, $\varepsilon_{t}$ représente le terme d'erreur qui est indépendant et uniformément distribué. La variable TPM mesure le niveau de développement du secteur des télécommunications au Bénin et représente les télécommunications dans ce modèle. À la suite des travaux antérieures (Roller et Waveman, 2001; Chakraborty et Nandi, 2003) et surtout compte tenu de la croissance explosive des communications mobiles au cours des dernières années, le taux de pénétration mobile est utilisé comme indicateur de mesure du niveau du développement des télécommunications. De même, s'inspirant de la littérature notamment (Youssef et M'Henni, 2004 ; Chabossou, 2017), nous utilisons le taux brut de scolarisation secondaire (TBSS) variable proxy du capital humain et l'investissement dans les TIC (INVTIC) représentant le stock de capital investi dans les TIC comme variables de contrôle.

Nos données proviennent de World Development Indicators (WDI, 2017), de World Telecommunications Indicators (WTI, 2017) et de l'Institut National de la Statistique, de l'Analyse Economique (INSAE, 2017) et elles couvrent la période 1980-2017.

Pour l'estimation économétrique, la nature des variables nous amène à recourir aux techniques de l'économétrie des séries temporelles à savoir l'analyse de la stationnarité des séries, l'analyse d'éventuelles relations de cointégration entre les séries et l'analyse de la causalité. Ces analyses nous permettrons de choisir la spécification appropriée (VAR, ECM ou VECM). Ainsi, si les variables sont stationnaires en niveau, on procède à l'estimation d'un modèle VAR (vector autoregression) en niveau ; si les variables sont stationnaires en première différence et qu'il existe de relation de cointégration entre ces dernières, on procède à l'estimation d'un modèle à correction d'erreur ou error correction model (ECM) ; si les variables sont stationnaires en différence première et qu'il n'existe pas de relation de cointégration, on estime un VAR en différence première ; si les variables sont stationnaires en différents ordres d'intégration, on procède à l'estimation d'un VAR en harmonisant (c'est-à-dire en ramenant à un même ordre d'intégration) ces différences d'ordre d'intégration.

\section{4- Présentations et analyses des résultats}

\section{4-1- Test de racine unitaire}

Pour les tests de racine unitaire, nous utilisons les tests standards de Dickey-Fuller augmenté (ADF) sur nos variables avec les valeurs critiques de Mckinnon disponibles pour déterminer l'ordre d'intégration des séries temporelles individuelles. Les tests standards ADF ont pour hypothèse nulle, la non stationnarité de la série, c'est-à-dire la présence d'une racine unitaire 
dans la série étudiée. Les tests de non stationnarité ADF sont effectués selon une procédure séquentielle en trois étapes. Le test de racine unitaire, à partir du test standard de Dickey-Fuller Augmenté donne les résultats qui sont présentés dans le tableau 1.

Tableau 1 : Présentation des résultats du test ADF en niveau et en différence première au seuil de $5 \%$

\begin{tabular}{|l|l|l|l|l|}
\hline \multirow{2}{*}{ Variables } & \multicolumn{2}{l|}{ En niveau } & \multicolumn{2}{l|}{ En différence première } \\
\cline { 2 - 5 } & ADF & Valeur critique & ADF & Valeur critique \\
& Statistique & Mckinnon & Statistique & Mckinnon \\
\hline LPIB & -0.226707 & -2.951125 & -3.671458 & -2.951125 \\
\hline LINVTIC & 2.257890 & -2.943427 & -4.383551 & -2.945842 \\
\hline LTBSS & 0.122097 & -2.943427 & -8.842398 & -2.945842 \\
\hline LTPM & -2.025194 & -2.945842 & -3.909768 & -2.945842 \\
\hline
\end{tabular}

Source : Compulation de l'auteur à partir des résultats des tests d'ADF

Au seuil fixé de 5\%, les valeurs critiques des différentes variables sont inférieures aux valeurs de l'ADF statistique en niveau. On accepte l'hypothèse nulle de la présence de racine unitaire pour toutes les séries. Donc la production nationale (LPIB), le taux brut de scolarisation secondaire (LTBSS), le taux de pénétration mobile (LTPM) et l'investissement dans le TIC (LINVTIC) ne sont pas stationnaires. Par contre, en différence première, toutes les séries du modèle sont stationnaires, car les valeurs critiques du test de l'ADF sont supérieures aux valeurs statistiques de l'ADF au seuil de 5\%. Toutes les séries sont donc intégrées d'ordre 1 au seuil de $5 \%$. On suspecte, selon la théorie économique, qu'il y a des relations économiques de long terme entre ces séries à niveau. Par conséquent, la présomption de cointégration révélée par les tests ADF ci-dessus réalisés mérite d'être infirmée ou confirmée par le test de cointégration de Johansen basé sur le critère de la trace.

\section{4-2- Test de cointégration de Johansen}

Nous utilisons le test de cointégration de Johansen qui nous permet de déterminer le nombre de relations d'équilibre de long terme entre des variables intégrées quel que soit l'ordre et quelle que soit la normalisation utilisée. Ce test permet par la méthode de maximum de vraisemblance de tester l'existence d'une relation de long terme dans les séries temporelles intégrées et d'obtenir tous les vecteurs de cointégration dans un cadre multivarié. L'étude de cointégration de Johansen présente les résultats de deux tests : le test de trace et le test de la valeur propre maximale. Lorsque les deux tests donnent des conclusions différentes, on retient en général les résultats du test de trace dont la puissance est plus élevée que celle du test de la valeur propre maximale (Cadoret et al., 2009). Pour notre analyse, nous retenons le test de trace de 
cointégration. Le tableau 2 présente les résultats du test de trace de cointégration.

Tableau n²: Test de la trace sur les variables logarithmiques

\begin{tabular}{|c|c|c|c|}
\hline Valeurs propres & Hypothèses nulles & Traces statistiques & Seuil critique à 1 \% \\
\hline 0.996051 & $\mathrm{r}=0$ & 149.3700 & 76.07 \\
\hline 0.947068 & $\mathrm{r}=1$ & 56.89128 & 58.46 \\
\hline 0.737797 & $\mathrm{r}=2$ & 30.74872 & 35.65 \\
\hline 0.573065 & $\mathrm{r}=3$ & 12.00780 & 20.04 \\
\hline 0.006556 & $\mathrm{r}=4$ & 0.092084 & 6.65 \\
\hline
\end{tabular}

Source : Compulation de l'auteur à partir des sorties du logiciel

L'analyse des résultats contenus dans le tableau ci-dessus révèle que la statistique de Johansen relative à la première valeur propre est supérieure au seuil de $1 \%(149.3700>76.07)$ à sa valeur critique ; on rejette alors l'hypothèse nulle d'absence de cointégration $(\mathrm{r}=0)$ au seuil statistique de $1 \%$. En revanche, on accepte l'hypothèse nulle $(\mathrm{r}=1)$ selon laquelle il existe une relation de cointégration entre les variables logarithmiques du modèle (58.46 $>56.89128$ d'après la 3è ligne du tableau). Les variables de notre modèle sont donc cointégrées au seuil de 5\%. Autrement dit, ces variables suivent des évolutions parallèles sur la période de 1980 à 2017. D'où la nécessité de réaliser un modèle à correction d'erreur. Pour l'estimation du modèle à correction d'erreur, il existe deux méthodes : le modèle à correction d'erreur à deux étapes d'Engle et Granger et le modèle à correction d'erreur en une seule étape de Hendry. Nous adoptons la méthode d'Engle et Granger qui revient dans un premier temps à identifier les relations de cointégration, en estimant par la méthode des moindres carrés ordinaires des relations de long terme entre les variables. Dans un second temps, des relations de court terme entre les variables, prises en différence première, sont estimées par la méthode des moindres carrés ordinaires.

\section{4-3- Estimation de la relation de long terme}

L'estimation de la dynamique de long terme est celle qui ressort de la spécification du modèle. Les résultats de l'estimation de la relation de long terme entre la production et les variables explicatives par la méthode des moindres carrés ordinaires sont présentés dans le tableau 3 suivant : 
Tableau 3 : Résultat de l'estimation de la relation de long terme (voir annexe)

\begin{tabular}{|l|l|l|l|}
\hline Variable expliquée : LPIB \\
\hline Variables explicatives & Coefficient & t-statistic & Probabilité \\
\hline C & 0.577142 & 0.335013 & 0.7397 \\
\hline LINVTIC & 0.581674 & 3.911347 & $0.0004 * * *$ \\
\hline LTBSS & 0.283928 & 1.767653 & $0.0861^{*}$ \\
\hline LTPM & 0.255516 & 1.276097 & 0.2106 \\
\hline $\begin{array}{l}\mathrm{R}^{2}=0.815996 \\
\text { Prob(F) }=0.000000\end{array}$ & $\mathrm{DW}=2.137631$ & $\mathrm{~F}($ Stat $)=50.25954$ \\
\hline
\end{tabular}

Source : Compulation de l'auteur à partir des résultats de l'estimation du modèle de long terme

*** coefficients significatifs à $1 \%$;** coefficients significatifs à $5 \%$; *coefficients significatifs à $10 \%$

Le coefficient de détermination $\left(\mathrm{R}^{2}=0.815996\right)$ et la statistique du test de Fisher indiquent une bonne adéquation du modèle et montrent que les variables exogènes de notre modèle expliquent à 81,5996\% les fluctuations observées au niveau de la production nationale. Il est alors possible d'estimer le modèle à correction d'erreur qui permet d'analyser la dynamique de court terme entre les variables.

\section{4-4- Résultats de l'estimation de la dynamique de court terme:}

L'étude des comportements de court terme repose sur l'estimation par les MCO du modèle à correction d'erreur avec le terme d'erreur retardée d'une période. Les résultats de l'estimation de la relation de court terme sont présentés dans le tableau 4. Ces résultats sont obtenus grâce à l'estimation du modèle à correction d'erreur des dynamiques de court terme représentées par les variables en différence première par la méthode des moindres carrés ordinaires.

Tableau 4 : Résultat de l'estimation de la dynamique de court terme (MCE)

\begin{tabular}{|c|c|c|c|}
\hline \multicolumn{4}{|c|}{ Variable expliquée : D(LPIB) } \\
\hline Variables explicatives & Coefficient & t-statistic & Probabilité \\
\hline $\mathrm{C}$ & 1.384316 & 1.742129 & $0.0911 *$ \\
\hline D(LINVTIC) & 0.174075 & 0.282309 & 0.7795 \\
\hline $\mathrm{D}(\mathrm{LTBSS})$ & 0.021564 & 0.131369 & 0.8963 \\
\hline $\mathrm{D}(\mathrm{LTPM})$ & 0.047221 & 2.153431 & $0.0090 *$ \\
\hline RESIDUS(-1) & -0.183695 & -2.685075 & $0.0007 * * *$ \\
\hline $\begin{array}{l}\mathrm{R}^{2}=0.793684 \\
\operatorname{Prob}(\mathrm{F})=0.000001\end{array}$ & $\mathrm{DW}=2.805355$ & & $=0.826946$ \\
\hline
\end{tabular}

Source : Réalisé par les auteurs à partir du modèle de court terme.

*** coefficients significatifs à $1 \% ; * *$ coefficients significatifs à $5 \%$; *oefficients significatifs à $10 \%$

$R^{2}=0.793684$; cette valeur étant supérieure à $50 \%$, l'ajustement linéaire est de bonne qualité. Le coefficient du résidu retardé, qui représente 
la force de rappel vers l'équilibre, est négatif (-0.183695) statistiquement significatif. De plus sa valeur est comprise entre -1 et 0 . La représentation du modèle à correction d'erreur est donc validée.

Le coefficient de détermination et la statistique du test de Fisher montrent que le modèle est globalement significatif. Avant d'interpréter ce résultat, il est indispensable d'étudier la qualité de l'estimation à l'aide des tests de robustesse des résidus. Pour la normalité, le test de Jarque-Bera permet de conclure que les résidus sont normalement distribués. En ce qui concerne l'hétéroscédasticité des erreurs, le test de White montre que les résidus sont homosscédastiques. Tous ces tests concourent à la validation du modèle estimé dont on peut à présent interpréter les résultats.

\section{4-5- Analyse des résultats}

En ce qui concerne le modèle de court terme, le coefficient associé au résidu de l'équation de la relation de long terme, retardé d'un an, est négatif et significativement différent de 0 à $1 \%$ (le modèle à correction d'erreur est donc satisfaisant) confirmant ainsi l'existence d'un mécanisme à correction d'erreur : à long terme, les déséquilibres entre l'évolution de la production et celles du taux brut de scolarisation secondaire (TBSS), du stock de capital investi dans les TIC (INVTIC) et du taux de pénétration mobile (TPM) se compensent de sorte que les variables ont des évolutions similaires sur la période de l'étude. Le coefficient de correction d'erreur indique la vitesse à laquelle tout déséquilibre entre les niveaux désiré et effectif de la production nationale est résorbé dans l'année qui suit le choc, la valeur 0,183695 représente donc la vitesse à laquelle le déséquilibre est résorbé. Ainsi, un choc sur la production nationale au Bénin au cours d'une année est entièrement résorbé au bout de $\left(\frac{1}{0,183695}\right.$ années $=5,44$ années $)$ soit au bout de cinq ans et 5 mois.

Le coefficient de la variable taux de pénétration (TPM) est significatif car sa probabilité est inférieure à $10 \%$, ce qui justifie qu'à court terme la variable TPM a une influence significative sur le PIB réel. Puisque la variable TPM affiche un coefficient positif et significatif alors une augmentation du niveau de pénétration mobile de $10 \%$ entrainerait une augmentation de la production totale de $0,47221 \%$. Ce résultat met en exergue l'importance de la contribution du taux de pénétration mobile dans la croissance du PIB réel au Bénin. Ce résultat indique qu'un accroissement du nombre d'abonnés locaux à la ligne téléphonique fixe et au téléphone mobile se traduirait par une amélioration du niveau de développement du secteur des télécommunications ce qui aura pour conséquence de booster la production nationale.

Le modèle montre, qu'à long terme, le coefficient de la variable investissement en TIC est positif et significatif au seuil de $1 \%$. La valeur de 
l'élasticité de long terme de l'investissement en TIC par rapport à la production nationale s'établit à 0,581674 . Ainsi, une augmentation de $10 \%$ de l'investissement en TIC entraine une augmentation de 5,8\% de la production nationale. La valeur relativement faible de cette élasticité indique que le Bénin ne bénéficie pas encore totalement du fort potentiel des TIC à tirer la croissance de la production nationale. Ce résultat est conforme à ceux de Youssef et M'Henni (2004) qui ont conclu à l'existence d'une relation positive entre les investissements TIC et la croissance économique en Tunisie. Ce résultat corrobore également les conclusions de certains travaux notamment (Cronin et al., 1991 ; Gary et Scott 2000 ; Anusua et Sumit, 2004 ; Chabossou, 2017).

Quant à la variable taux brut de scolarisation secondaire (TBSS), elle affiche un coefficient positif et significatif à long terme. D'où cette variable exerce une influence positive sur le PIB réel au Bénin. Comme le postule la théorie économique, le capital humain est l'un des facteurs importants de la croissance de la production nationale et de sa qualité. Les résultats obtenus dans cette étude montrent que dans le cas du Bénin cette conclusion théorique trouve une justification à long terme. Le capital humain apparait comme l'une des principales sources du développement économique du Bénin.

\section{4-6- Télécommunications et croissance économique : une analyse de la causalité au sens de Granger}

L'existence d'un rapport de corrélation entre deux variables n'indique pas nécessairement qu'il existe un rapport de causalité entre elles. En effet, la détermination du sens de causalité est importante et a des implications en matière de politique économique. Ainsi, pour améliorer les résultats de l'analyse de la relation entre les télécommunications et la croissance de l'économie du Bénin, l'étude de la causalité entre le taux de pénétration mobile (TPM) et la production à travers la mise en œuvre du test de causalité de Granger est envisagée. L'objectif est de savoir si le développement accéléré du secteur des télécommunications a amené la causalité dans un sens où ce sont les télécommunications qui causent la croissance économique d'un point de vue statistique. Autrement dit, dans un cadre de prévision, est-on arrivé à un stade où il serait économiquement plus intéressant de prédire le niveau de la croissance économique en se basant sur les valeurs courantes et passées de la croissance dans l'accumulation du capital technologique.

L'hypothèse nulle du test effectué est « la variable X ne cause pas la variable $Y$ au sens de Granger $»$. Une p-value suffisamment faible par rapport au niveau de confiance choisi conduit au rejet de l'hypothèse nulle. Le niveau de confiance que nous retenons ici est $5 \%$. Le tableau 5 présente les résultats du test de causalité de Granger qui est retenu. 
Tableau 5 : Test de causalité de Granger

\begin{tabular}{|c|c|c|c|}
\hline \multicolumn{2}{|c|}{$\begin{array}{l}\text { Hypothèse nulle (X ne cause pas } \\
\text { Y) }\end{array}$} & \multirow{2}{*}{ P-value obtenue } & \multirow[t]{2}{*}{$\begin{array}{l}X \text { cause t'il } Y \text { au sens de } \\
\text { Granger? }\end{array}$} \\
\hline X & Y & & \\
\hline PIB & TPM & 0.0243 & Oui \\
\hline TPM & PIB & 0.0452 & Oui \\
\hline
\end{tabular}

Source : Compulation de l'auteur à partir des résultats du test de causalité

Plusieurs théories économiques relatent une probable relation de causalité entre le PIB et le taux de pénétration mobile des télécommunications. Il s'agit de procéder à la vérification de cette probable existence de causalité dans le cas du Bénin. C'est dans ce cadre que le test de Granger a été effectué pour vérifier cette théorie dans le cas du Bénin.

Les résultats du test de causalité de Granger révèlent l'existence d'une causalité bidirectionnelle entre le taux de pénétration mobile dans le secteur des télécommunications et la croissance économique. Le résultat du test de causalité montre que la causalité va dans les deux sens : du taux de pénétration mobile vers la production nationale et vice-versa au seuil de 5\%. Il est possible de prédire le niveau de la croissance économique en connaissant la croissance du niveau de pénétration mobile. De même, une amélioration du niveau de croissance constitue une opportunité d'amélioration du niveau de diffusion des TIC au Bénin. Ce résultat est encourageant pour les réformes en cours dans le secteur des télécommunications au Bénin.

\section{Conclusion}

L'importance des Technologies de l'Information et de la Communication (TIC) dans le développement économique rapide que l'Afrique connait actuellement est bien connue. Ainsi, l'investissement dans les TIC à travers l'amélioration de l'accès aux réseaux de téléphonie créée des opportunités d'emploi, de transfert de connaissance et une meilleur efficacité et transparence en matière économique. Les TIC accroissent les possibilités des pays en développement et plus particulièrement le Bénin de participer aux marchés internationaux. Par ailleurs, l'étude de la relation de corrélation entre télécommunication et croissance économique qui a été l'objet ce travail a permis d'abord de faire une brève présentation de l'évolution du secteur des télécommunications au Bénin. Ensuite, une synthèse de la littérature sur le lien entre télécommunication et croissance a permis de montrer que le débat sur ce lien penche pour l'existence d'un lien positif. Enfin, le recours à la modélisation économétrique des séries temporelles basée sur la méthode de correction d'erreur à la Engle et Granger a permis de tester d'une part l'hypothèse de corrélation positive, et d'autre part celle de causalité entre les télécommunications et la croissance économique au Bénin. Sur la base des 
estimations, l'étude a abouti à la conclusion que non seulement les télécommunications influencent positivement et significativement la croissance économique, mais aussi leur lien de causalité est bidirectionnel, alors l'usage de la téléphonie mobile améliore la croissance économique et vice versa et de ce fait l'existence d'un réseau de télécommunication performant et accessible constitue pour l'économie nationale, un facteur de la croissance économique. De plus, au regard de la contribution positive de l'investissement liés aux télécommunications sur la croissance, il est légitime que le gouvernement béninois renforce cette catégorie de dépenses, afin de créer un cadre favorable aux activités des entreprises, et par conséquent à la croissance. Enfin, en utilisant efficacement les télécommunications, les entreprises peuvent réaliser des gains de productivités qui renforcent leur compétitivité et contribuent ainsi à une croissance économique durable, une condition de réduction de la pauvreté.

\section{References:}

1. Alleman J., Hunt C., Michaels D., Mueller M., Rappoport P., and Taylor L. (2014) : Telecommunications and Economic Development: Empirical Evidence from Southern Africa; International Telecommunications Society, Sydney.Available from http://www.colorado.edu/engineering /alleman /print_ files/soafrica paper.pdf.

2. Anusua D. and Sumit A. (2004) Telecommunications and economic growth: a panel data approach, Applied Economics, 36:15, 1649-1654, DOI: $10.1080 / 0003684042000218552$

3. ARCEP-BENIN (2017) : « Rapport annuel d'activités 2017 », Bénin, 84p, Décembre 2017.

4. ARCEP-BENIN (2018). Observation de la téléphonie mobile au Bénin, Rapport général.

5. Arlandis J. (1987). «Le développement des télécommunications : les enjeux économiques de la mutation des années $80 »$, Revue TiersMonde, tome 28, $\mathrm{n}^{\circ} 111$, pp. 535-542.

6. ATCI (2009) : Les externalités de réseaux et le développement des télécommunications dans les pays en développement; Séminaire régional sur les coûts et tarifs pour le groupe régional pour l'Afrique de la commission d'étude 3 (SG3RG-AFR); MAPUTO DU 4 AU 8 MAI 2009.

7. Banque mondiale (1994): «Rapport sur le développement dans le monde: une infrastructure pour le développement», Presse de l'université de Washington. 
8. Barro, R. J. (1990). Government spending in a simple model of endogeneous growth. Journal of Political Economy, 98 (S5), pp.103125.

9. Cadoret I. ; Benjamin C. ; Martin F. ; Herrard N. et Tanguy S. (2009) : «Econométrie appliquée : Méthodes - applications - corrigés ». 2e édition. Edition De Boeck, Coll. Ouvertures Economiques.

10. Chabossou A. F. (2010) : Revue de Performance du Secteur Des TIC Benin, Towards Evidence-based ICT Policy and Regulation Volume Two, Policy Paper 18, 2010/

11. Chabossou, A. F. (2015). "Fixed and mobile telephones in WAEMU countries: complementary or substitute services?". Journal of African Studies and Development (JASD). Vol. 7(11), pp. 256-263,

12. Chabossou, A. F. (2017) «Effets des technologies de l'information et de la communication sur la croissance économique du Bénin», Revue "Repères et Perspectives Economiques" [En ligne], 02 / 2ème semestre 2017, mis en ligne le 28 décembre 2017.

13. Chakraborty, C. and B. Nandi, 2003. Privatization, telecommunications and growth in selected Asian countries: An econometric analysis. Communications and Strategies, 52: 31-47.

14. Cieslika, A. and M. Kaniewsk, 2004. Telecommunications infrastructure and regional economic development. The Case of Poland, Regional Studies, 38: 713-725.

15. Cronin J.F., E. B. Parker, E. K. Colleran, and M. A. (1991): "Telecommunications infrastructure and economic growth: an analysis of causality"; Telecommunications policy, pp529-535.

16. Cronin, F.J., E.K. Colleran, P.L. Herbert and S. Lewitzky, 1993. Telecommunications and growth: The contribution of telecommunications infrastructure investment to aggregate and sectoral productivity. Telecommunications Policy, 17: 677-690.

17. Economic Commission for Africa (ECA) (1999). Politiques et Stratégies de pour accélérer le développement de l'infrastucture de l'information en Afrique, Addis Abeba, ECA.

18. Gary M. and Scott J. S. (2000) : Telecommunications and economic growth; International Journal of Social Economics, Vol. 27 No. 7/8/9/10, 2000, pp. 893-906.

19. Greenstein, S. and P.T. Spiller, 1996. Estimating the welfare effects of digital infrastructure, Working Paper No. 5770. Cambridge, MA.: National Bureau of Economic Research.

20. GSMA (2017): ''L'économie du secteur mobile: Afrique subsaharienne", Rapport général. 
21. Hardy, A.P., (1980). "The role of the telephone in economic development', Telecommunications Policy, Volume 4, Issue 4, pp 278-286.

22. INSAE (2010): Les entreprises des TIC au Bénin, Rapport thématique ; deuxième recensement général des entreprises (RGE 2)

23. Jipp, A., 1963. Wealth of nations and telephone density. Telecommunications Journal, 30: 199-201

24. Karner, J. and R. Onyeji, 2007. Telecom private investment and economic growth: The case of African and central \& East European countries. Unpublished Thesis, Jonkoping University, Jonkoping International Business School, JIBS, Economics.

25. Lichtenberg, F., 1995. The output contributions of computer equipment and personnel: A firm-level analysis. Economics of Innovation and New Technology, 3: 201-217.

26. Madden, G. and S.J. Savage, 1998. CEE telecommunications investment and economic growth. Information Economics and Policy, 10: 173-195.

27. Meleu M. (1999) : « Participation du secteur privé à la fourniture des infrastructures en Côte d'Ivoire : Revue et recommandation »; CIRES, No 47, Economic Research Papers, Université d'Abidjan.

28. Moss, M.L., 1981. Telecommunications and productivity. MA: Addison-Wesley.

29. Qiang, C.Z.W., 2009. What are the economic and social impacts of the mobile phone sector in developing countries? Propaco's MagazineIssue: 7-9.

30. Ricketts, M., 2002. The economics of business enterprise. $U K$ : Cheltenham: Edward Elgar.

31. Röller L. H. and Waverman L. (2001): "Telecommunications Infrastructure and Economic Development: A Simultaneous Approach', The American Economic Review, Vol. 91, No. 4 (Sep., 2001), pp. 909-923

32. Romer M. P. (1986) : 'Increasing Returns and Long-Run Growth', Journal of Political Economy, Vol. 94, No. 5, pp. 1002-1037.

33. Saunders, R.J., J.J. Warford and B. Wellenius, 1994. Telecommunications and economic development. Baltimore: The Johns Hopkins University Press.

34. SCRP (2016): Evaluation de la stratégie de croissance pour la réduction de la pauvreté (SCRP 2011-2015), Rapport final, Bénin, 118 , Décembre 2016

35. Shiu, A. and P.L. Lam, 2008. Causal Relationship between Telecommunications and Economic Growth in China and its Regions, Regional Studies, 42:5, 705-718 
36. Solow, R. M. (1956) "A Contribution to the Theory of Economic Growth." Quarterly Journal of Economics, 70(1), pp. 65-94.

37. Sridhar, K. S. et Sridhar, V. (2007): Telecommunications infrastructure and economic growth: evidence from developing countries; Applied Econometrics and International Development, Vol.7-2 (2007)

38. Waverman, L., M. Meschi and M.A. Fuss, 2005. The impact of telecoms on economic growth in developing countries, in the Vodafone Policy Paper Series 3. Vodafone: 10-24.

39. Wolde-Rufael, Y., 2007. Another look at the relationship between telecommunications investment and economic activity in the United States. International Economic Journal, 21: 199-205.

40. Yoo, S.H. and S.J. Kwak, 2004. Information technology and economic development in Korea: A causality study. International Journal of Technology Management, 27: 57-67.

41. Youssef A. B. et M'Henni M. (2004) : « Les effets des TIC sur la croissance économique : le cas de la Tunisie », Revue Région et Développement $\mathrm{n}^{\circ} 19-2004$.

\section{Annexes}

\section{Annexe 1 : TEST DE RACINE UNITAIRE}

PIB

\section{A NIVEAU}

Null Hypothesis : PIB has a unit root

Exogenous: Constant

Lag Length: 3 (Automatic - based on SIC, maxlag=9)

\begin{tabular}{llll}
\hline \hline & t-Statistic & Prob.* \\
\hline \hline Augmented Dickey-Fuller test statistic & -0.226707 & 0.9255 \\
\hline Test critical values: & 1\% level & -3.639407 & \\
& 5\% level & -2.951125 & \\
& 10\% level & -2.614300 & \\
\hline \hline
\end{tabular}

*MacKinnon (1996) one-sided p-values. 


\section{EN DIFFERENCE PREMIERE}

Null Hypothesis : D(PIB) has a unit root

Exogenous : Constant

Lag Length : 2 (Automatic - based on SIC, maxlag=9)

\begin{tabular}{llll}
\hline \hline & t-Statistic & Prob.* \\
\hline \hline Augmented Dickey-Fuller test statistic & -3.671458 & 0.0001 \\
\hline Test critical values: & 1\% level & -3.639407 & \\
& 5\% level & -2.951125 & \\
& 10\% level & -2.614300 & \\
\hline \hline
\end{tabular}

*MacKinnon (1996) one-sided p-values.

\section{INVTIC \\ A NIVEAU}

Null Hypothesis : INVTIC has a unit root

Exogenous: Constant

Lag Length : 0 (Automatic - based on SIC, maxlag=9)

\begin{tabular}{llll}
\hline \hline & t-Statistic & Prob. $^{*}$ \\
\hline \hline Augmented Dickey-Fuller test statistic & 2.257890 & 0.9999 \\
\hline Test critical values: & 1\% level & -3.621023 & \\
& 5\% level & -2.943427 & \\
& 10\% level & -2.610263 & \\
\hline \hline
\end{tabular}

*MacKinnon (1996) one-sided p-values.

\section{EN DIFFERENCE PREMIERE}

Null Hypothesis: D(INVTIC) has a unit root

Exogenous: Constant

Lag Length: 0 (Automatic - based on SIC, maxlag=9)

\begin{tabular}{llll}
\hline \hline & t-Statistic & Prob. $^{*}$ \\
\hline \hline Augmented Dickey-Fuller test statistic & -4.383551 & 0.0013 \\
\hline Test critical values: & 1\% level & -3.626784 & \\
& 5\% level & -2.945842 & \\
& 10\% level & -2.611531 & \\
\hline \hline
\end{tabular}

*MacKinnon (1996) one-sided p-values. 


\section{TBSS}

\section{A NIVEAU}

Null Hypothesis : TBSS has a unit root

Exogenous : Constant

Lag Length: 0 (Automatic - based on SIC, maxlag=9)

\begin{tabular}{llll}
\hline \hline & t-Statistic & Prob.* \\
\hline \hline Augmented Dickey-Fuller test statistic & 0.122097 & 0.9632 \\
\hline Test critical values: & 1\% level & -3.621023 & \\
& 5\% level & -2.943427 & \\
& 10\% level & -2.610263 & \\
\hline \hline
\end{tabular}

*MacKinnon (1996) one-sided p-values.

EN DIFFERENCE PREMIERE

Null Hypothesis : D(TBSS) has a unit root

Exogenous : Constant

Lag Length : 0 (Automatic - based on SIC, maxlag=9)

\begin{tabular}{llcl}
\hline \hline & t-Statistic & Prob. $^{*}$ \\
\hline \hline Augmented Dickey-Fuller test statistic & -8.842398 & 0.0000 \\
\hline Test critical values: & 1\% level & -3.626784 & \\
& 5\% level & -2.945842 & \\
& $10 \%$ level & -2.611531 & \\
\hline \hline
\end{tabular}

*MacKinnon (1996) one-sided p-values.

\section{TPM}

\section{A NIVEAU}

Null Hypothesis : TPM has a unit root

Exogenous : Constant

Lag Length : 1 (Automatic - based on SIC, maxlag=9)

\begin{tabular}{llll}
\hline \hline & t-Statistic & Prob.* \\
\hline \hline Augmented Dickey-Fuller test statistic & -2.025194 & 0.2752 \\
\hline Test critical values: & 1\% level & -3.626784 & \\
& 5\% level & -2.945842 & \\
& 10\% level & -2.611531 & \\
\hline \hline
\end{tabular}

*MacKinnon (1996) one-sided p-values. 


\section{EN DIFFERENCE PREMIERE}

Null Hypothesis : D (TPM) has a unit root

Exogenous : Constant

Lag Length : 0 (Automatic - based on SIC, maxlag=9)

\begin{tabular}{llll}
\hline \hline & t-Statistic & Prob.* \\
\hline \hline Augmented Dickey-Fuller test statistic & -3.909768 & 0.0048 \\
\hline Test critical values: & 1\% level & -3.626784 & \\
& 5\% level & -2.945842 & \\
& 10\% level & -2.611531 & \\
\hline \hline
\end{tabular}

*MacKinnon (1996) one-sided p-values.

Annexe 2: ESTIMATION DU MODELE DE LONG TERME

Dependent Variable: LPIB

Method: Least Squares

Date: $12 / 22 / 18$ Time: 11:28

Sample: 19802017

Included observations: 38

\begin{tabular}{lllll}
\hline \hline Variable & Coefficient & Std. Error & t-Statistic & Prob. \\
\hline \hline C & 0.577142 & 1.722746 & 0.335013 & 0.7397 \\
LINVTIC & 0.581674 & 0.148714 & 3.911347 & 0.0004 \\
LTBSS & 0.283928 & 0.160624 & 1.767653 & 0.0861 \\
LTPM & 0.255516 & 0.200232 & 1.276097 & 0.2106 \\
\hline \hline R-squared & 0.815996 & Mean dependent var & 7.262556 \\
Adjusted R-squared & 0.799760 & S.D. dependent var & 1.090844 \\
S.E. of regression & 0.488132 & Akaike info criterion & 1.502840 \\
Sum squared resid & 8.101288 & Schwarz criterion & 1.675218 \\
Log likelihood & -24.55396 & Hannan-Quinn criter. & 1.564171 \\
F-statistic & 50.25954 & Durbin-Watson stat & 2.137631 \\
Prob(F-statistic) & 0.000000 & & \\
\hline \hline
\end{tabular}




\section{Annexe 4: DYNAMIQUE DE COURT TERME}

Dependent Variable: D(LPIB)

Method: Least Squares

Date : 12/22/18 Time : 11:30

Sample (adjusted) : 19812017

Included observations : 37 after adjustments

\begin{tabular}{lllll}
\hline \hline Variable & Coefficient & Std. Error & t-Statistic & Prob. \\
\hline \hline C & 1.384316 & 0.794612 & 1.742129 & 0.0911 \\
D(LINVTIC) & 0.174075 & 0.616611 & 0.282309 & 0.7795 \\
D(LTBSS $)$ & 0.021564 & 0.164148 & 0.131369 & 0.8963 \\
D(LTPM) & 0.047221 & 0.307770 & 2.153431 & 0.0090 \\
RESIDUS(-1) & -0.183695 & 0.109013 & -2.685075 & 0.0007 \\
\hline \hline
\end{tabular}

\begin{tabular}{llll} 
R-squared & 0.793684 & Mean dependent var & 0.077168 \\
Adjusted R-squared & 0.019605 & S.D. dependent var & 0.668208 \\
S.E. of regression & 0.674726 & Akaike info criterion & 2.176069 \\
Sum squared resid & 14.56817 & Schwarz criterion & 2.393760 \\
Log likelihood & -35.25727 & Hannan-Quinn criter. & 2.252815 \\
F-statistic & 0.826946 & Durbin-Watson stat & 2.805355 \\
Prob(F-statistic) & 0.000001 & & \\
\hline
\end{tabular}

Annexe 5 : TEST D'HETEROSCEDASTICITE

Heteroskedasticity Test: Breusch-Pagan-Godfrey

\begin{tabular}{llll}
\hline \hline F-statistic & 2.074948 & Prob. F(4,32) & 0.1073 \\
Obs*R-squared & 7.620196 & Prob. Chi-Square(4) & 0.1065 \\
Scaled explained SS & 45.63660 & Prob. Chi-Square(4) & 0.0000 \\
\hline \hline
\end{tabular}

Annexe 6: TEST D'AUTOCORRELATION DES ERREURS

Breusch-Godfrey Serial Correlation LM Test:

\begin{tabular}{llll}
\hline \hline F-statistic & 1.459190 & Prob. F(2,19) & 0.2573 \\
Obs*R-squared & 3.728134 & Prob. Chi-Square(2) & 0.1550 \\
\hline \hline
\end{tabular}


Annexe 7 : TEST DE JARQUE-BERA

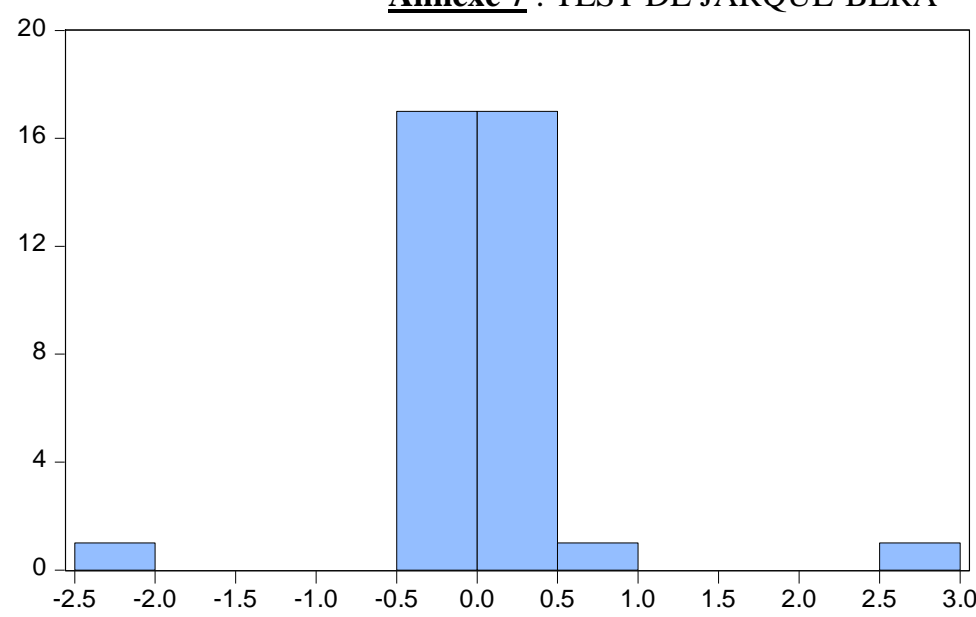

Series: Residuals

Sample 19812017

Observations 37

Mean

$1.65 \mathrm{e}-16$

Median $\quad 0.038440$

Maximum $\quad 2.963734$

Minimum $\quad-2.122234$

Std. Dev. $\quad 0.636138$

Skewness $\quad 1.801998$

Kurtosis

17.01329

Jarque-Bera $\quad 3.765200$

Probability $\quad 0.232323$ 